\title{
Variability of the global electron content during the major sudden stratospheric warmings in January 2009
}

\author{
A.V. Timchenko ${ }^{1}$, F.S. Bessarab ${ }^{1}$, M.V. Klimenko ${ }^{1}$, V. V. Klimenko ${ }^{1}$, \\ E. V. Rozanov' ${ }^{1}$, T. V. Sukhodolov' ${ }^{1}$, O. P. Borchevkina' ${ }^{1}$ Y. N. Korenkov', \\ N. A. Korenkova ${ }^{1}$, K. G. Ratovsky ${ }^{2}$ \\ ${ }^{1}$ West Department of Pushkov Institute of Terrestrial Magnetism, Ionosphere and \\ Radio Wave Propagation Russian Academy of Sciences, Kaliningrad, 236035, Russia \\ ${ }^{2}$ Institute of Solar-Terrestrial Physics Siberian Branch, Russian Academy of Sciences, \\ Irkutsk, 664033, Russia \\ E-mail: timchenko.leks@gmail.com
}

\begin{abstract}
The paper presents the investigation of the Global Electron Content (GEC) variability during a major Sudden Stratospheric Warming (SSW) event in January 2009. The two-hour values of the GEC obtained from the GIM, as well as the GEC calculated in the EAGLE and GSM TIP models, were used as initial data. It has been shown that during SSW event the amplitude of diurnal variations in GEC decreases and the amplitude of semidiurnal variations increases. GEC variability during SSW event increases, as well as during the small geomagnetic disturbance that occurred on January 26, 2009.
\end{abstract}

Accepted: 15.09 .2020

DOI: 10.21046/2070-7401-2020-17-6-167-172

\section{Introduction}

The Earth's ionosphere is affected by a complex of physical and chemical processes that lead to fluctuations in the ionospheric parameters concerning a certain background state.

Most of the ionospheric variability can be explained by such powerful control processes as the solar radiation flux and geomagnetic activity. However, a significant part of the ionospheric variability (about $20 \%$ ) is associated with processes in the lower atmosphere [1]. Thus, an understanding of the atmosphere-ionosphere coupling is great importance for predicting the state of the ionosphere and a deeper understanding of the ionosphere plasma physics. Sudden Stratospheric Warming (SSW) event is a dramatic meteorological phenomenon in the high-latitude stratosphere, the response of which is observed in the parameters of the thermosphere-ionosphere system [2].

In this paper, we investigated the response of the Global Electron Content (GEC) to major SSW in January 2009.

The GEC represents the total number of electrons in the Earth's ionosphere. The methodology for calculating GEC based on Global Ionosphere Maps (GIM) [3] was proposed by prof. E. L. Afraimovich [4]. Since the calculation is based on GIM data obtained using data from Global Navigation Satellite Systems (GNSS) the GEC includes the total number of electrons from the Earth up to heights of $\sim 20200 \mathrm{~km}$. The temporary resolution of GIM (and, accordingly, GEC) is from 2 hours to 15 minutes. Previous studies [4] showed that regular diurnal, 27-day, semi-annual and annual variations are distinguished in the GEC time series. In general, GEC can be considered as recognized integral characteristic of the ionosphere. 
This paper presents the results of a study of GEC variations in January 2009, including period of major 2009 SSW.

\section{Initial data and research methods}

In this paper, we consider the ionospheric variability in January 2009 to study its relationship with major 2009 SSW event. The development of this famous SSW event is shown using daily average temperature variations in polar latitudes $\left(60-90^{\circ} \mathrm{N}\right)$ at a pressure level $10 \mathrm{hPa}(\sim 31 \mathrm{~km})$ according to NCEP/NCAR reanalysis temperature data [5] (figure 1). The maximum temperature deviation from the average value for the considered period reached $35 \mathrm{~K}$. The presented temperature variation almost perfectly corresponds to the theoretical determination of the temperature dynamics in the stratosphere during the SSW events. This warming is concern to the major SSW event due to significant temperature changes and when the winter polar stratospheric westerlies reverse to easterly.

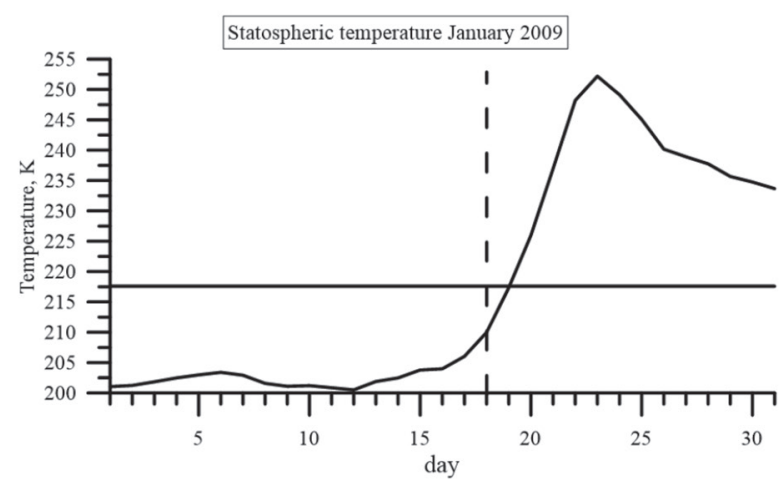

Figure 1. Variation of daily mean stratospheric temperature at a height of $10 \mathrm{hPa}$. The horizontal line indicates the average value for the period from January 1 to January 31, 2009. A vertical dashed line indicates the onset day of an SSW event.

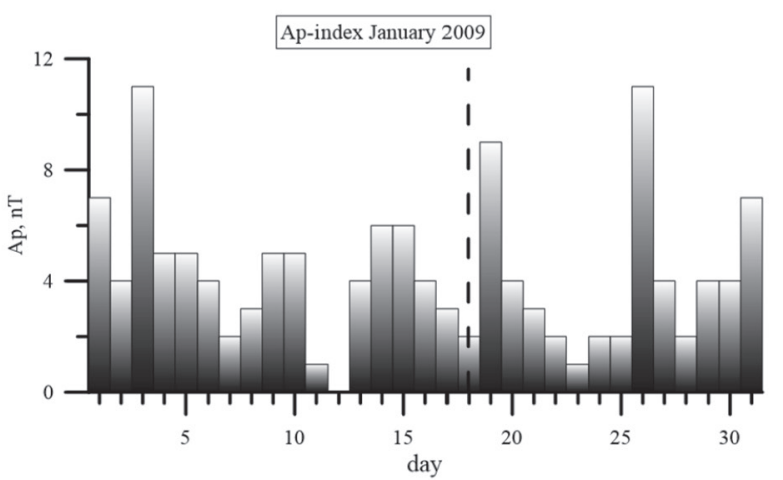

Figure 2. Dynamics of the daily average $A_{p}$-index in January 2009. A vertical dashed line indicates the onset day of an SSW event.

The SSW-2009 event is unique, as it occurred against the background of abnormally low solar and geomagnetic activities (the value of the F10.7 index averaged over the considered period was only 71 s.f.u.). The dynamics of the Ap-index from January 1 to January 31, 2009, are shown in figure 2. The diagram shows that the Ap-index did not exceed the value of $18 \mathrm{nT}$ for the entire period.

To analyse the GEC temporal variations, we used the two-hour data of the Global Electron Content IGS (International GNSS Service) products, available at https://simurg.iszf.irk.ru/.

The study of the observed GEC variations was carried out in comparison with the model values of GEC, which were obtained in the calculations of the Entire Atmosphere Global Model (EAGLE) and the Global Self-consistent Model of the Thermosphere, Ionosphere, Protonosphere (GSM TIP).

Calculations of the thermosphere-ionosphere system during selected periods were made with the first principles GSM TIP developed in WD IZMIRAN [6]. The model gives time-dependent global three-dimensional distributions of the neutral upper atmosphere parameters (temperature, composition and wind velocity vectors) from 80 to $526 \mathrm{~km}$, the ionosphere parameters (density, temperature, and velocity vectors of atomic and molecular ions and electrons) from $80 \mathrm{~km}$ up to 15 Earth radii, the electric field potential of thermospheric and magnetospheric origin.

The EAGLE model [7] combines the Hamburg Model of the Neutral and Ionized Atmosphere (HAMMONIA) model and the upper atmosphere model GSM TIP.

In EAGLE model run the SSW event was setting using reanalysis data. During model experiments, the geomagnetic conditions were assumed unchanged. Thus, the resulting perturbations of the ionosphere were caused only by dynamic processes generated by atmosphere-ionosphere coupling. The statement of the problem for EAGLE simulation of the January 2009 is described in detail by M. V. Klimenko et al. [7]. In GSM TIP model run the geomagnetic conditions were assumed to be unchanged; only the day-to-day variability in the solar zenith angle reflecting the internal seasonal variability of the ionosphere were taking into account. 
In figure 3 presented the variations of the GEC based on observational data (figure $3 a$ ) and the results of calculations of the EAGLE (figure 3b) and GSM TIP (figure 3c) models.
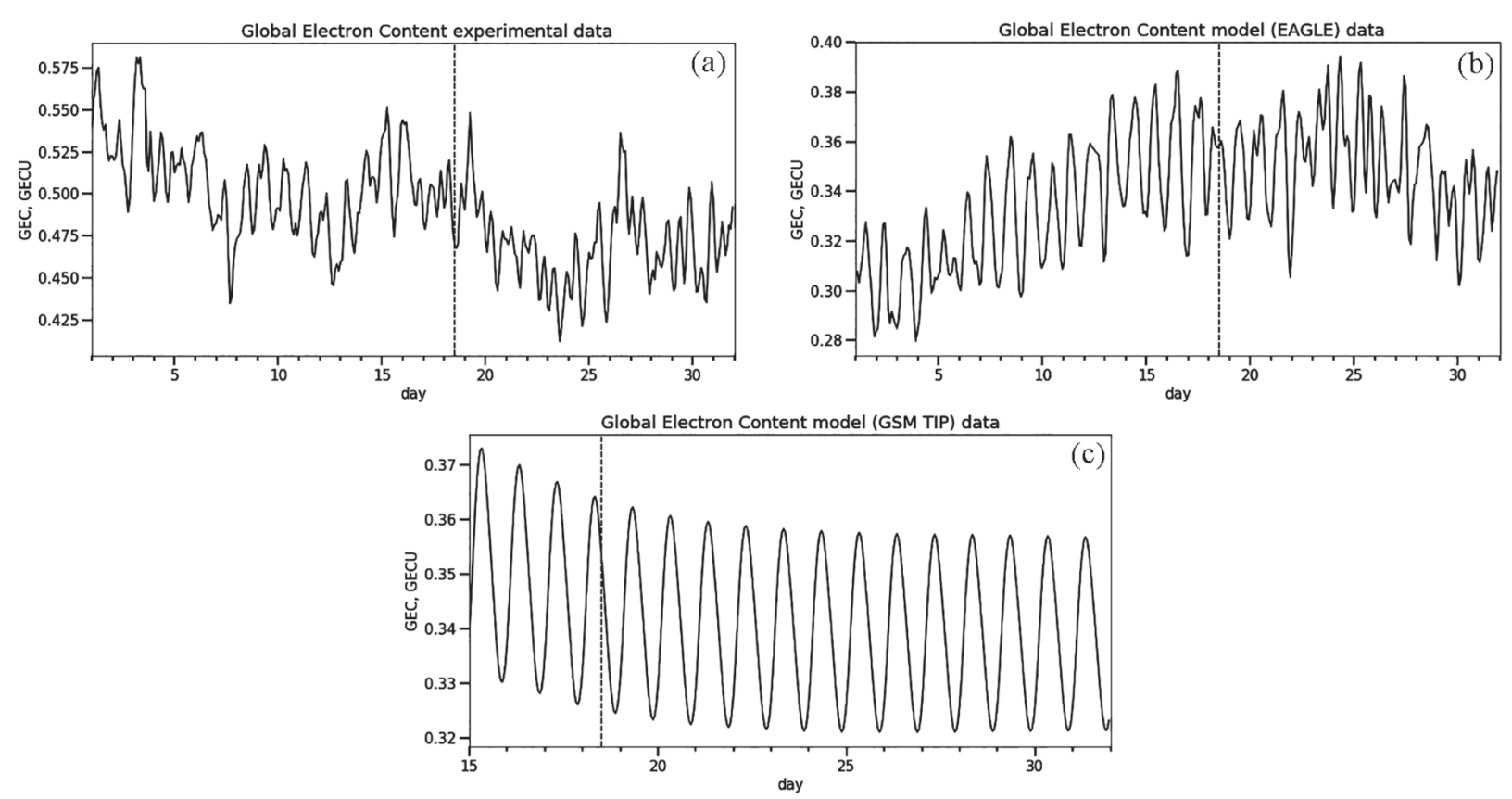

Figure 3. The behaviour of the Global Electron Content in January 2009 according to observations (a) and according to the calculation results of the EAGLE (b) and GSM TIP (c) models. A vertical dashed line indicates the onset day of an SSW event.

A preliminary analysis of GEC variations performed using the continuous wavelet transform $W(a, b)$ :

$$
W(a, b)=\frac{1}{\sqrt{|a|}} \int_{-\infty}^{\infty} f(t) \psi *\left(\frac{t-b}{a}\right) \mathrm{d} t,
$$

where $f(t)$ is the GEC variations, $\psi(t)$ is the Morlet wavelet function, parameter $a$ is the scale, $b$ is the time shift, $t$ is time, ${ }^{*}$ denotes complex conjugation.

Wavelet Morlet has the following form:

$$
\psi(t)=\exp (i \pi t) \cdot \exp \left(-t^{2} / 0.7^{2}\right)
$$

It is convenient to represent the complex coefficients of the Morlet wavelet function (2) calculated by (1) as the sum of the squares of their real and imaginary parts:

$$
E_{a, b}=\operatorname{Re}(W(a, b))^{2}+\operatorname{Im}(W(a, b))^{2} .
$$

In this representation, $E_{a, b}$ has the meaning of spectral energy.

It is known that during SSW event dramatic changes occur in the dynamics of the high-latitude stratosphere, which leads to a significant increase in stochastic oscillations in ionospheric parameters. To assess the variability of ionospheric parameters during the SSW event, we used the value of the standard deviation of the GEC diurnal variation from the monthly average diurnal variation, which was calculated for each day by the formula:

$$
S_{d}=\left(\frac{1}{N} * \sum_{i=1}^{N}\left(x_{d, i}-\overline{X_{i}}\right)^{2}\right)^{1 / 2},
$$

where $d$ is the day number, $i$ is the measurement number during the day, $N$ is the number of measurements per day, $\overline{X_{i}}$ is the average monthly value of the parameter for the $i$-th hour, $x_{d, i}$ is the $i$-th measurement for $d$-day. 


\section{Results and discussion}

Figure 4 shows the squares of the GEC wavelet coefficients calculated by (3) for January 2009. The dashed line indicates the beginning of the SSW event. It can be seen that during SSW the amplitude of diurnal harmonics decreases and the amplitude of semidiurnal harmonics in GEC variations increases. A similar effect is also seen in the results of the GEC wavelet analysis obtained from the EAGLE model results. However, there is a slight delay in this effect - weakening of the diurnal and amplification of the semidiurnal harmonics - in the GEC variations according to the EAGLE results relative to the observational data. Also, in the observational data, a distinct peak is seen in the daily harmonics around January 26, which is absent in the model results. This peak is probably related to the substorm that occurred on that day, the effects of which was not taken into account in model runs.
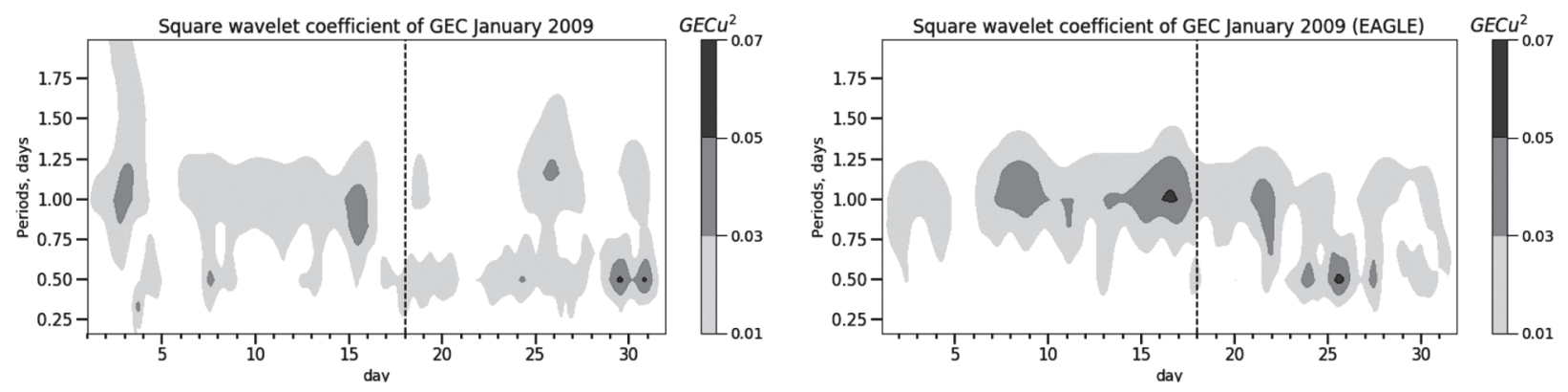

Figure 4. Squares of wavelet GEC coefficients for January 2009. A vertical dashed line indicates the onset day of an SSW event.

We estimated the day-to-day GEC variability based on the calculation results of the GSM TIP and EAGLE models and according to observation data in January 2009. For this purpose, the standard deviations of the GEC were calculated by (4) for each day. The results of this calculation are presented in figure 5.

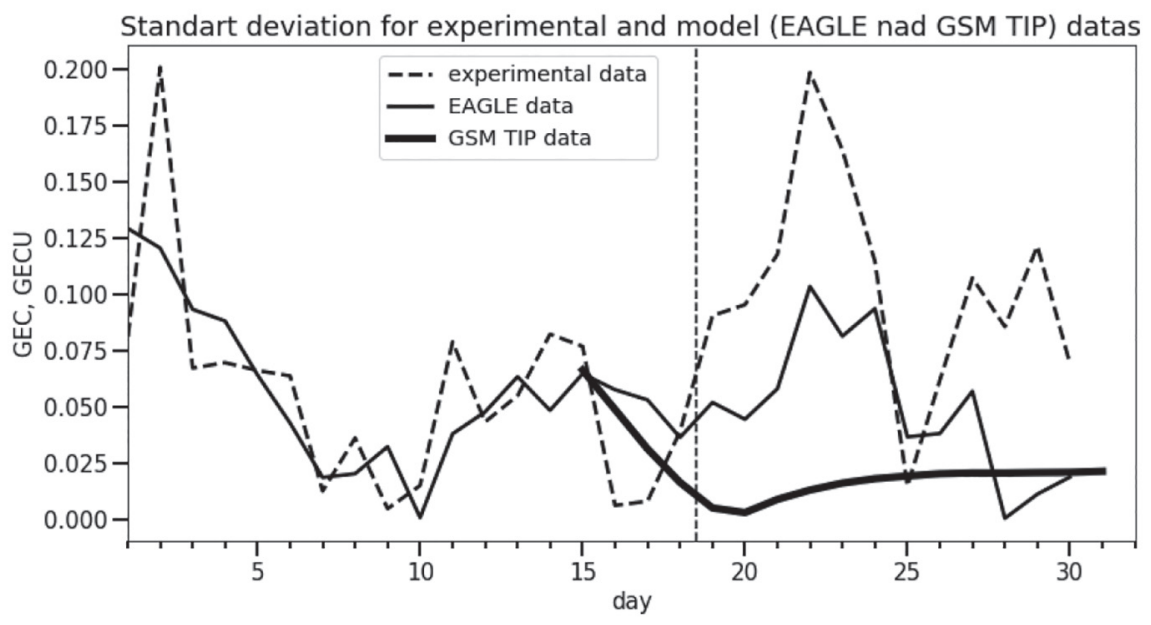

Figure 5. The standard deviation of the Global Electron Content based on observational data (dashed line) and model results (EAGLE (solid thin line) and GSM TIP (solid bold line)) for January 2009. A vertical dashed line indicates the onset day of an SSW event.

From the figure 5, one can see that the variability according to the observational data has the largest amplitude. Peaks of variability according to the EAGLE model and according to observational data occur: at the beginning of January; during period of January 11-15; and at period of the SSW temperature peak (January 20-24). As mentioned earlier, the geomagnetic substorm was not taken into account in the EAGLE model; therefore, the peak of variability formed in observational data near January 26 is not reproduced in EAGLE model results. As for GSM TIP, its results do not indicate the formation of any observed features, which is explained by the statement of the problem used in GSM TIP. 
As an additional analysis, we calculated the standard deviation for the variations in the critical frequency of the ionospheric F2-layer (foF2) over Irkutsk and Kaliningrad according to ionosonde data and the EAGLE and GSM TIP model simulations. The variability of foF2 are shown in figure 6.
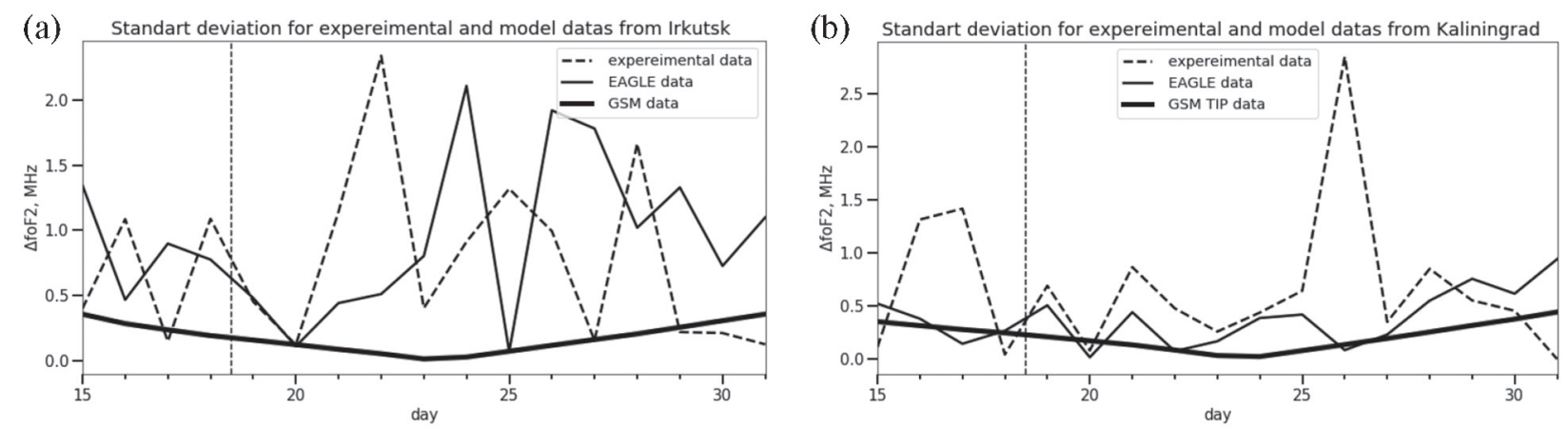

Figure 6. The standard deviation of the variations in the critical frequency of the ionosphere F2 layer according to the observational data (dashed line) and calculation results for the EAGLE (solid thin line) and GSM TIP (solid bold line) models over (a) Irkutsk and (b) Kaliningrad for 15-31 January 2009. A vertical dashed line indicates the onset day of an SSW event.

As can be seen, the variability of the foF2 over Irkutsk is higher than over Kaliningrad. The results of the calculations of the EAGLE model are in good agreement with observations, especially over Irkutsk. The increase in foF2 variability over Irkutsk is clearly seen during the passage of SSW (January 21-25). However, the variability of foF2 according to the EAGLE model during the SSW period has a small delay of about 3-4 days, relative to the variability according to observations. The variability of foF2 over Kaliningrad does not reveal a significant association with the SSW event. Over Kaliningrad, in the observational data, there are two peaks of foF2 variability, which are absent in EAGLE model results. We associate the second peak with the geomagnetic substorm on January 26, and the reason for the first peak is still unknown. As for the results of GSM TIP calculations, the formation of any observed features was not revealed for foF2 over Irkutsk and Kaliningrad, as for GEC. The EAGLE model provides significantly greater foF2 variability compared to GSM TIP, and in the case of relative disturbances, the variability according to the EAGLE model coincides with the observed one.

\section{Conclusion}

Our investigation shows that the EAGLE model reproduces the following observed features of the ionospheric response to the major 2009 SSW event:

1. During the SSW event, the amplitude of diurnal harmonics of the GEC variations reduced and the semi-diurnal harmonic's amplitude increased.

2. GEC and foF2 variabilities over Irkutsk increased, but foF2 variability over Kaliningrad remained stable during the SSW event.

It should be noted that during the geomagnetic substorm on January 26, 2009, according to observation data, the daily harmonic in GEC variations does not reduce, but on the contrary, increase. The GEC variability increases, as well as during the major SSW event. The variability of foF2 over Kaliningrad on January 26 also increases.

\section{Acknowledgements}

The reported study was funded by Russian Foundation for Basic Research (Project No. 18-05-00594).

\section{References}

[1] Forbes J. F., Palo S. E., Variability of the ionosphere, J. Atmospheric and Solar-Terrestrial Physics, 2000, Vol. 62, pp. 685-693.

[2] Chau J.L., Goncharenko L.P., Fejer B. G., Liu H.-L., Equatorial and low latitude ionospheric effects during sudden stratospheric warming events ionospheric effects during SSW events, Space Science Reviews, 2012, Vol. 168(1-4), pp. 385-417. 
[3] Afraimovich E. L., Astafyeva E. I., Oinats A. V., Yasukevich Y. V., Zhivetiev I. V., Global electron content: a new conception to track solar activity, Annals Geophysics, 2008, Vol. 26, pp. 335-344.

[4] Hernández-Pajares M., Juan J. M., Sanz J., Orus R., Garcia-Rigo A., Feltens J., Komjathy A., Schaer S. C., Krankowski A., The IGS VTEC maps: a reliable source of ionospheric information since 1998, J Geodesy, 2009, Vol. 83(3-4), pp. 263-275.

[5] Kalnay E., Kanamitsu M., Kistler R., Collins W., Deaven D., Gandin L., Iredell M., Saha S., White G., Woollen J., Zhu Y., Chelliah M., Ebisuzaki W., Higgins W., Janowiak J., Mo K. C., Ropelewski C., Wang J., Leetmaa A., Reynolds R., Roy J., Joseph D., The NCEP/NCAR 40-year reanalysis project, Bull. American Meteorological Society, 1996, Vol. 77, pp. 437-471.

[6] Korenkov Y. N., Klimenko V. V., Forster M., Bessarab F. S., Surotkin V.A., Calculated and observed ionospheric parameters for a Magion 2 passage and EISCAT data on July 31, 1990, J. Geophysical Research, 1998, Vol. 103(A7), pp. 14697-14710.

[7] Klimenko M. V., Klimenko V.V., Bessarab F. S., Sukhodolov T. V., Vasilev P.A., Karpov I. V., Korenkov Y. N., Zakharenkova I. E., Funke B., Rozanov E. V., Identification of the mechanisms responsible for anomalies in the tropical lower thermosphere/ionosphere caused by the January 2009 sudden stratospheric warming, J. Space Weather Space Climate, 2019, Vol. 9, A39, 14 p. 